\title{
DIFFERENTIAL DISTRIBUTION OF PDE4B SPLICE VARIANT mRNAS IN RAT BRAIN AND THE EFFECTS OF SYSTEMIC ADMINISTRATION OF LPS IN THEIR EXPRESSION
}

Elisabet Reyes-Irisarri ${ }^{1}$, Silvia Pérez-Torres ${ }^{1}$, Xavier Miró $^{2}$, Emili Martínez $^{1}$, Pere

Puigdomènech ${ }^{2}$, José M. Palacios ${ }^{3}$, Guadalupe Mengod ${ }^{1}$.

1: Dept. of Neurochemistry, Institut d'Investigacions Biomèdiques de Barcelona, CSIC (IDIBAPS).

2: Dept. of Molecular Genetics, Institut de Biologia Molecular de Barcelona, CSIC.

3: Parc Cientìic, Universitat de Barcelona.

Running title: PDE4B splice variants expression in rat brain

Correspondence to: Guadalupe Mengod. Dept. of Neurochemistry, Institut d'Investigacions Biomèdiques de Barcelona, CSIC (IDIBAPS). Rosselló, 161, 6ª 08036 Barcelona, Spain.Tel 34 9336383 23, fax 349336383 01, e-mail: gmInqr@iibb.csic.es

Keywords: CAMP, inflammation, PDE4B splicing variant, in situ hybridization 
The phosphodiesterase 4 (PDE4) family, which specifically hydrolyzes intracellular CAMP, is composed by four isozymes (PDE4A, PDE4B, PDE4C and PDE4D) encoded by different gene loci, and each of them produces several mRNAs by alternative splicing (Houslay et al., 1998). These isozymes and their splice variants are widely expressed in many tissues including the brain (Pérez-Torres et al., 2000; McPhee et al., 2001; Miró et al., 2002; D'Sa et al., 2005). The different knock out mice for PDE4B and PDE4D genes present unique phenotypes, (Jin et al., 1999; Jin and Conti, 2002; Jin et al., 2005), which suggests different functions for these isozymes.

The majority of studies into PDE4 subtype distribution have focused upon inflammatory cells (Engels et al., 1994; Uhlig et al., 1997; Gantner et al., 1998). PDE4A, PDE4B and PDE4D, are found to some extent in different inflammatory cell types, where they could be important regulators of inflammatory processes. Selective inhibitors of PDE4s have been suggested as therapies for the treatment of several human diseases (Menniti et al., 2006), predominantly disorders of the immune and inflammatory systems (Teixeira et al., 1997) or disorders of the central nervous system, such as, depression (O'Donnell and Zhang, 2004) or Alzheimer's disease (McGeer and McGeer, 1995).

The lipopolysaccharide (LPS) is a potent inflammatory agent that has been used to characterize the acute inflammatory process in the brain (Breder et al., 1994; Breder and Saper, 1996). The efficacy of PDE4 inhibitors in inflammation models is remarkable and includes inhibition of LPS-induced increment in serum levels of tumour necrosis factor- $\alpha$ (TNF- $\alpha$ ) in liver injury, lung injury, renal failure, mortality (Teixeira et al., 1997; Jin and Conti, 2002) or in a multiple sclerosis mouse model (Moore et al., 2006).

The present study aims to determine the regional distribution in rat brain of the four PDE4B splice variants. The possible involvement of CAMP through PDE4B in inflammation prompted us to analyze the expression of the different PDE4 isozymes in the brain of LPS treated rats.

\section{MATERIALS AND METHODS}

Animal procedure was performed according to the European Union regulations (O.J. of E.C. L358/1 18/12/1986) and was approved by the Institutional Animal Care and Use Committee. Adult male Wistar rats (200-300 g; Iffa Credo, Lyon, France) used for the neuroanatomical localization of PDE4B splicing variants expression were decapitated and the brain was removed, frozen on dry ice and kept at $-20^{\circ} \mathrm{C}$. LPS treatment was performed as follows: rats were injected intraperitoneally with vehicle or LPS (from Escherichia coli, serotype 055:B5, Sigma, St. Louis, MO, USA; $500 \mu \mathrm{g} / \mathrm{kg}$ of body weight) diluted in $300 \mu \mathrm{l}$ of $0.9 \%$ sterile saline. Four rats were sacrificed at each of 1, 2, 3 and $4 \mathrm{hr}$ post injection. Eight control rats received an injection of $0.9 \%$ sterile saline, and were sacrificed four at each of 2 and $3 \mathrm{hr}$ post injection.

Several oligonucleotides were synthesized for each splicing variant: PDE4B1, bp 383-427, and 506-550, AF202732; PDE4B2 bp 418-462, 520-564, 545-589, L27058; PDE4B3 bp 476-520, 556-600, 616-660, 700-744, U95748; PDE4B4 bp 171-215, 216-260, 264-308, AF202733. COX-2 mRNA oligonucleotides were complementary to bp 1848-1893, NM_017232. The mRNA regions for each $\mathrm{PDE} 4 \mathrm{~B}$ splice variants were chosen because they share no similarity with each 
other. Evaluation of the oligonucleotide sequences with basic local alignment search tool of EMBL and GenBank databases indicated that the probes do not present any significant similarity with mRNAs other than their corresponding targets in the rat. The specificity of the autoradiographic signal obtained in the in situ hybridization histochemistry experiments was confirmed by performing a series of routine controls (Pompeiano et al., 1992) such as the use of different oligonucleotides for the same mRNA obtaining identical hybridization patterns with each individual probe in the entire rat brain; competition with the same unlabeled oligonucleotide for the non-specific hybridization signal, determination of the $T_{m}$ of the hybrids, etc. The procedures followed for the in situ hybridization experiments have been published elsewhere (Reyes-Irisarri et al., 2005). The results presented in this work have been done using only one of the different oligonucleotides designed for each splicing form appropriately labeled at their 3 ' end with ${ }^{33} \mathrm{P}-\mathrm{dATP}$.

A semiquantitative measure of the optical densities of the autoradiograms was conducted with the MCID4 and $\mathrm{AIS}^{\mathrm{R}}$ computerized image analysis systems (Imaging Research Inc, St Catharines, Ontario, Canada). Anatomical brain structures were verified by the examination of cresyl violet-stained sections and identified using a rat brain atlas (Paxinos and Watson, 1998). For each rat, individual values of optical densities in each region were calculated as the mean of 2 adjacent sections in both hemispheres (average of 4 measures per rat and region). The following regions were measured: area postrema, CA fields of the hippocampus, cerebellum, choroid plexus, corpus callosum, dorsal cochlear nucleus, dentate gyrus, hilus, leptomeninges, medial genicular nucleus, medial habenular nucleus, median eminence, organum vasculosum of the lamina terminalis, primary somatosensory cortex, pyramidal tract. Statistical analysis was performed using GraphPad Prism 4 (GraphPad Software Inc, San Diego, CA, USA) and SPSS v14 (SPSS Inc, Chicago, IL, USA). Analysis of data variance for all the experiments were performed considering the factors PDE (4 levels), brain region (13 levels) and treatment (5 levels: control and 1, 2, 3 and 4 hours post injection). Then, the different regions were analyzed by factors PDE and treatment. Post hoc analysis (Bonferroni's test) was performed for treatment by each PDE in the statistically affected regions. Since no significant statistical difference between the two groups of control rats was observed in any of the regions analyzed, we unified the values as a single control group.

\section{RESULTS AND DISCUSSION \\ mRNA distribution of PDE4B splicing variants in rat brain}

We used in situ hybridization histochemistry to study the distribution of the four PDE4B splicing variants at various coronal levels of the rat brain (Fig. 1). Table I summarizes our semiquantitative measurements of the content of each mRNA in different brain regions. The splicing variants identified by hybridization with only one oligonucleotide each (and allways the same) show a coincident hybridization pattern in several brain areas, such as granular layer of the cerebellum and piriform cortex. In contrast they are differentially expressed in other brain areas, indicating a possible non-redundant role in the regulation of intracellular CAMP levels. 
Interestingly, PDE4B1, PDE4B2 and PDE4B3 are detected in the area postrema whereas PDE4B4 is absent (or expressed at very low levels). This area has been implicated as a chemoreceptor trigger zone for emesis (Borinson H.L. and Wang, 1953; Carpenter et al., 1988). The emetic side effects of rolipram are well known, when used as an antidepressant, (Scott et al., 1991). So too are those that other PDE4 inhibitors (such as roflumilast and cilomilast) produce in patients treated with them for asthma or chronic obstructive pulmonary disease (COPD) (Spina, 2003; Chung, 2006). The fact that we found no expression of PDE4B4 mRNA in the rat area postrema (even though rodents do not vomit) and that it has been proposed that in humans PDE4B4 exon is unlikely to encode a protein (Shepherd et al., 2003), points to the importance of determining the expression of PDE4 splice variants in this area and other related areas of the human brain. This will help to find candidate targets for specific splicing variant PDE4 inhibitors with no emetic side effects to treat the aforementioned diseases.

In the hippocampal formation all four isoforms are visualized in CA2 and CA3 fields. In the dentate gyrus the only splicing isoform found was PDE4B2. The basic circuitry of the hippocampus is a trisynaptic circuit (Brown and Zador, 1990) where the sensory inputs from the entorhinal cortex arrive at the granule cells of the dentate gyrus, whose main output -via mossy fiber axons- is the pyramidal neurons of CA3, that send their Schaffer collateral axons to pyramidal neurons of CA1. Some of the synapses of the hippocampus display a remarkable form of plasticity, long-term potentiation (LTP), that could be relevant to the mnemonic functions of this circuitry (Brown et al., 1988), and is a leading candidate for a synaptic mechanism for rapid learning in mammals (Brown and Zador, 1990). This form of plasticity has been studied most in the mossy fiber and in the Schaffer collateral axons. Several authors (Barad et al., 1998; Bach et al., 1999; Rose et al., 2005) suggest that rolipram could enhance hippocampusdependent memory tasks, and reduce cognitive decline associated with neurodegenerative and psychiatric diseases. These studies together with our finding that PDE4B2 is the only splicing variant expressed in the dentate gyrus, suggest that it could be involved in LTP regulation in the mossy fiber axons. The findings of Ahmed and Frey (2003) that PDE4B3 is involved in the regulation of LTP in the hippocampus, do not invalidate our suggestion, since there are different mechanisms for the two LTP systems: the mossy fibers and the Schaffer collateral inputs. PDE4B3 could be involved in the LTP of Schaffer collateral inputs, (which take place in the CA3 region where all the splicing variants of PDE4B are expressed) and PDE4B2, could be involved in the LTP system of the mossy fibers output, which originates in the dentate gyrus, where PDE4B2 is the only splicing form expressed.

\section{Systemic administration of LPS: effects on the brain.}

In order to study the effects of short-term systemic administration of LPS on the expression of PDE4 isozymes in rat brain, we measured the corresponding mRNA levels 1, 2, 3 and $4 \mathrm{hr}$ after LPS treatment in 15 brain areas (including some circumventricular organs) for the 4 splicing variant mRNAs. In order to validate our inflammatory animal model, we analyzed COX2 mRNA expression in LPS-brains, since COX-2 mRNA is up-regulated in endothelial and perivascular cells of rat and mouse brain blood vessels after LPS administration (Cao et al., 
1997). As expected, we found that COX-2 mRNA levels were considerably increased $2 \mathrm{hr}$ after LPS injection in microvasculature and leptomeninges (Fig. 2). The analysis of the autoradiogram optical densities for the four PDE4B splicing variant mRNAs indicated a strong influence of the factors PDE $(F=126.750 ; p<0.0001)$ and region $(F=337.929 ; p<0.0001)$, while the influence of treatment was indicated by the interaction between PDE-treatment $(F=2.057$; $p<0.05)$. Analysis of variance for factors PDE and treatment at the different regions identifies OVTL, hilus, and choroid plexus as the regions where treatment effects were more consistent. Finally, Bonferroni's test performed for each PDE in these regions showed an exclusively significant increase in mRNA expression of PDE4B2 in choroid plexus when comparing vehicle with 2 and 3 hours after LPS injection $(p<0.01)$. In these animal groups mRNA presented a tendency to increase in the rest of the areas.

Upregulation in the expression of several mRNAs has been reported to occur in some of the brain areas we have measured, such as choroid plexus, leptomeninges, and some circumventricular organs (organum vasculosum of the lamina terminalis, median eminence and area postrema) in inflammatory models. Several authors have observed an overexpression of CD14 (Lacroix et al., 1998), TNF- $\alpha$ (Nadeau and Rivest, 1999; Quan et al., 1999), COX-2 (Breder and Saper, 1996; Lacroix and Rivest, 1998) or IKappaB- $\alpha$ (Quan et al., 1997) mRNAs in these regions after a peripheral injection of LPS. The upregulation of PDE4B2 mRNA we observed in the choroid plexus and circumventricular organs after LPS injection could be of interest, given the fact that they may be the sites where communication between peripheral immune system and the brain takes place. The potential role played by PDE4B in inflammation is suported by the work of Conti's group done in PDE4B knockout mice (Jin and Conti, 2002; Jin et al., 2005). Those animals present a decreased responsiveness to LPS in many inflammatory cells: peripheral leukocytes and peritoneal macrophages are not able to secrete TNF- $\alpha$ after LPS stimulation.

To conclude, we have observed a differential distribution of the four splicing variants of PDE4B in the rat brain and a differential regulation for one of the PDE4B splicing variants, PDE4B2, in an inflammatory model. Additional experiments and the development of specific inhibitors for this isoform will help to elucidate its implication in the inflammatory reaction.

\section{ACKNOWLEDGEMENTS}

Supported by grants awarded by Fundació La Marató de TV3 (\#1017/97), and CICYT (SAF2003-02083, SAF2006-10243). S.P.-T. and X.M. have received a fellowship from CIRIT (Generalitat de Catalunya). We wish to acknowledge technical help from Rocío Martín. We thank Robin Rycroft for English corrections.

\section{References}

Ahmed T and Frey JU. 2003. Expression of the specific type IV phosphodiesterase gene PDE4B3 during different phases of long-term potentiation in single hippocampal slices of rats in vitro. Neuroscience 117:627-638. 
Bach ME, Barad M, Son H, Zhuo M, Lu YF, Shih R, Mansuy I, Hawkins RD, and Kandel ER. 1999. Age-related defects in spatial memory are correlated with defects in the late phase of hippocampal long-term potentiation in vitro and are attenuated by drugs that enhance the cAMP signaling pathway. Proc Natl Acad Sci U S A 96:5280-5285.

Barad M, Bourtchouladze R, Winder DG, Golan H, and Kandel E. 1998. Rolipram, a type IVspecific phosphodiesterase inhibitor, facilitates the establishment of long-lasting long-term potentiation and improves memory. Proc Natl Acad Sci U S A 95:15020-15025.

Borinson H.L. and Wang SC. 1953. Physiology and pharmacology of vomiting. Pharmacol Rev 5:193-230.

Breder CD, Hazuka C, Ghayur T, Klug C, Huginin M, Yasuda K, Teng M, and Saper CB. 1994. Regional induction of tumor necrosis factor alpha expression in the mouse brain after systemic lipopolysaccharide administration. Proc Natl Acad Sci U S A 91:11393-11397.

Breder CD and Saper CB. 1996. Expression of inducible cyclooxygenase mRNA in the mouse brain after systemic administration of bacterial lipopolysaccharide. Brain Res 713:64-69.

Brown TH, Chapman PF, Kairiss EW, and Keenan CL. 1988. Long-term synaptic potentiation. Science 242:724-728.

Brown TH and Zador AM. 1990. Hippocampus. In Gordon M.Shepherd, editor. The Synaptic Organization of the Brain. New York: Oxford University Press. p 346-388.

Cao C, Matsumura K, Yamagata K, and Watanabe Y. 1997. Involvement of cyclooxygenase-2 in LPS-induced fever and regulation of its mRNA by LPS in the rat brain. Am J Physiol 272:R1712-R1725.

Carpenter DO, Briggs DB, Knox AP, and Strominger N. 1988. Excitation of area postrema neurons by transmitters, peptides, and cyclic nucleotides. J Neurophysiol 59:358-369.

Chung KF. 2006. Phosphodiesterase inhibitors in airways disease. Eur J Pharmacol 533:110117.

D'Sa C, Eisch AJ, Bolger GB, and Duman RS. 2005. Differential expression and regulation of the cAMP-selective phosphodiesterase type $4 \mathrm{~A}$ splice variants in rat brain by chronic antidepressant administration. Eur J Neurosci 22:1463-1475.

Engels P, Fichtel K, and Lubbert H. 1994. Expression and regulation of human and rat phosphodiesterase type IV isogenes. FEBS Lett 350:291-295.

Gantner F, Gotz C, Gekeler V, Schudt C, Wendel A, and Hatzelmann A. 1998.

Phosphodiesterase profile of human $B$ lymphocytes from normal and atopic donors and the effects of PDE inhibition on B cell proliferation. Br J Pharmacol 123:1031-1038.

Houslay MD, Sullivan M, and Bolger GB. 1998. The multienzyme PDE4 cyclic adenosine monophosphate-specific phosphodiesterase family: intracellular targeting, regulation, and selective inhibition by compounds exerting anti-inflammatory and antidepressant actions. Adv Pharmacol 44:225-342.

Jin SL and Conti M. 2002. Induction of the cyclic nucleotide phosphodiesterase PDE4B is essential for LPS-activated TNF-alpha responses. Proc Natl Acad Sci U S A 99:7628-7633.

Jin SL, Lan L, Zoudilova M, and Conti M. 2005. Specific role of phosphodiesterase 4B in lipopolysaccharide-induced signaling in mouse macrophages. J Immunol 175:1523-1531.

Jin SL, Richard FJ, Kuo WP, D'Ercole AJ, and Conti M. 1999. Impaired growth and fertility of cAMP-specific phosphodiesterase PDE4D- deficient mice. Proc Natl Acad Sci U S A 96:11998-12003. 
Lacroix S, Feinstein D, and Rivest S. 1998. The bacterial endotoxin lipopolysaccharide has the ability to target the brain in upregulating its membrane CD14 receptor within specific cellular populations. Brain Pathol 8:625-640.

Lacroix S and Rivest S. 1998. Effect of acute systemic inflammatory response and cytokines on the transcription of the genes encoding cyclooxygenase enzymes (COX-1 and COX-2) in the rat brain. J Neurochem 70:452-466.

McGeer PL and McGeer EG. 1995. The inflammatory response system of brain: implications for therapy of Alzheimer and other neurodegenerative diseases. Brain Res Rev 21:195-218.

McPhee I, Cochran S, and Houslay MD. 2001. The novel long PDE4A10 cyclic AMP phosphodiesterase shows a pattern of expression within brain that is distinct from the long PDE4A5 and short PDE4A1 isoforms. Cell Signal 13:911-918.

Menniti FS, Faraci WS, and Schmidt CJ. 2006. Phosphodiesterases in the CNS: targets for drug development. Nat Rev Drug Discov 5:660-670.

Miró X, Pérez-Torres S, Puigdomènech P, Palacios JM, and Mengod G. 2002. Differential distribution of PDE4D splice variant mRNAs in rat brain suggests association with specific pathways and presynaptical localization. Synapse 45:259-269.

Moore CS, Owens T, Earl N, Frenette R, Styhler A, Nicholson DW, Mancini JA, Hebb AL, and Robertson GS. 2006. PERIPHERAL PDE4 INHIBITION PRODUCED BY L-826,141 PREVENTS EXPERIMENTAL AUTOIMMUNE ENCEPHALOMYELITIS. J Pharmacol Exp Ther.

Nadeau S and Rivest S. 1999. Regulation of the gene encoding tumor necrosis factor alpha (TNF-alpha) in the rat brain and pituitary in response in different models of systemic immune challenge. J Neuropathol Exp Neurol 58:61-77.

O'Donnell JM and Zhang HT. 2004. Antidepressant effects of inhibitors of cAMP phosphodiesterase (PDE4). Trends Pharmacol Sci 25:158-163.

Paxinos G and Watson C. 1998. The Rat Brain in stereotaxic coordinates. San Diego: Academic Press.

Pérez-Torres S, Miró X, Palacios JM, Cortés R, Puigdomènech P, and Mengod G. 2000. Phosphodiesterase type 4 isozymes expression in human brain examined by in situ hybridization histochemistry and $\left[{ }^{3} \mathrm{H}\right]$ rolipram binding autoradiography. Comparison with monkey and rat brain. J Chem Neuroanat 20:349-374.

Pompeiano M, Palacios JM, and Mengod G. 1992. Distribution and cellular localization of mRNA coding for 5-HT1A receptor in the rat brain: correlation with receptor binding. $\mathrm{J}$ Neurosci 12:440-453.

Quan N, Stern EL, Whiteside MB, and Herkenham M. 1999. Induction of pro-inflammatory cytokine mRNAs in the brain after peripheral injection of subseptic doses of lipopolysaccharide in the rat. J Neuroimmunol 93:72-80.

Quan N, Whiteside M, Kim L, and Herkenham M. 1997. Induction of inhibitory factor kappaBalpha mRNA in the central nervous system after peripheral lipopolysaccharide administration: an in situ hybridization histochemistry study in the rat. Proc Natl Acad Sci U S A 94:10985-10990.

Reyes-Irisarri E, Perez-Torres S, and Mengod G. 2005. Neuronal expression of cAMP-specific phosphodiesterase 7B mRNA in the rat brain. Neuroscience 132:1173-1185.

Rose GM, Hopper A, De Vivo M, and Tehim A. 2005. Phosphodiesterase inhibitors for cognitive enhancement. Curr Pharm Des 11:3329-3334. 
Scott Al, Perini AF, Shering PA, and Whalley LJ. 1991. In-patient major depression: is rolipram as effective as amitriptyline? Eur J Clin Pharmacol 40:127-129.

Shepherd M, McSorley T, Olsen AE, Johnston LA, Thomson NC, Baillie GS, Houslay MD, and Bolger GB. 2003. Molecular cloning and subcellular distribution of the novel PDE4B4 CAMPspecific phosphodiesterase isoform. Biochem J 370:429-438.

Spina D. 2003. Phosphodiesterase-4 inhibitors in the treatment of inflammatory lung disease. Drugs 63:2575-2594.

Teixeira MM, Gristwood RW, Cooper N, and Hellewell PG. 1997. Phosphodiesterase (PDE)4 inhibitors: anti-inflammatory drugs of the future? Trends Pharmacol Sci 18:164-171.

Uhlig S, Featherstone RL, Held HD, Nusing R, Schudt C, and Wendel A. 1997. Attenuation by phosphodiesterase inhibitors of lipopolysaccharide- induced thromboxane release and bronchoconstriction in rat lungs. J Pharmacol Exp Ther 283:1453-1459. 
Table I. Estimated densities of PDE4B splicing variable mRNAs in different regions of the rat brain

\begin{tabular}{|c|c|c|c|c|}
\hline Brain area & PDE4B1 & PDE4B2 & PDE4B3 & PDE4B4 \\
\hline \multicolumn{5}{|l|}{ Cortex } \\
\hline Parietal cortex & +/++ & + & + & +/++ \\
\hline Frontal cortex & $+/++$ & + & + & $+/++$ \\
\hline Cingulate cortex & $+/++$ & + & + & + \\
\hline Retrospenial cortex & +/++ & + & + & + \\
\hline Entorhinal cortex & $+/++$ & + & + & + \\
\hline \multicolumn{5}{|l|}{ Olfactory system } \\
\hline Anterior olfactory nucleus & $-1+$ & $+/++$ & $+/++$ & $+/++$ \\
\hline Olfactory tubercle & + & $+/++$ & +/++ & + \\
\hline Piriform cortex & $++/+++$ & ++ & ++ & ++ \\
\hline Islands of Calleja & + & + & + & + \\
\hline Islands of Calleja, major island & + & $-1+$ & + & $-1+$ \\
\hline \multicolumn{5}{|l|}{ Basal ganglia and related areas } \\
\hline Caudate-Putamen & +/++ & $-/+$ & + & $-1+$ \\
\hline \multicolumn{5}{|l|}{ Accumbens } \\
\hline Core & + & $-1+$ & $+/++$ & $-/+$ \\
\hline Shell & ++ & + & + & + \\
\hline \multicolumn{5}{|l|}{ Limbic areas } \\
\hline \multicolumn{5}{|l|}{ Ammon's horn } \\
\hline CA1 (pyramidal cell layer) & $+/++$ & ++ & + & + \\
\hline CA2 (pyramidal cell layer) & $+/++$ & ++ & ++ & ++ \\
\hline CA3 (pyramidal cell layer) & $+/++$ & ++ & ++ & +/++ \\
\hline Dentate gyrus & ++ & $++/+++$ & $-1+$ & - \\
\hline Hilus & + & + & +/++ & $-1+$ \\
\hline Subiculum & + & + & + & $-1+$ \\
\hline Pre, parasubiculum & + & + & + & $-1+$ \\
\hline Amygdala & $+/++$ & + & + & + \\
\hline Lateral septal nucleus & $+/++$ & - & - & $-/+$ \\
\hline Medial septal nucleus & $-1+$ & $-1+$ & + & + \\
\hline \multicolumn{5}{|l|}{ Thalamus and Hypothalamus } \\
\hline Medial habenular nucleus & $-1+$ & +/++ & ++ & +/++ \\
\hline Lateral habenular nucleus & $-1+$ & + & ++ & $+/++$ \\
\hline Paraventricular thalamic nucleus & - & - & ++ & $+/++$ \\
\hline Ventroposterior thalamic nuclei & _- & + & + & - \\
\hline Laterodorsal thalamic nucleus & - & + & + & - \\
\hline Mediodorsal thalamic nucleus & - & + & + & - \\
\hline Posterior thalamic nucleus group & - & + & + & - \\
\hline Zona incerta & + & $-1+$ & +/++ & $-/+$ \\
\hline Reticular thalamic nucleus & - & $-1+$ & + & - \\
\hline Dorsomedial hypothalamus & $+/++$ & $-/+$ & $-1+$ & - \\
\hline Ventromedial hypothalamus & $+/++$ & + & $-1+$ & - \\
\hline Arcuate nucleus & - & $++/+++$ & - & - \\
\hline Periventricular hypothalamic nucleus & - & + & - & - \\
\hline Medial geniculate nucleus & $-1+$ & + & +/++ & +/++ \\
\hline Internal capsule & - & + & $++/+++$ & ++ \\
\hline \multicolumn{5}{|l|}{ Brainstem } \\
\hline Superior colliculus & + & + & $+/++$ & + \\
\hline Oculomotor nucleus & - & + & $+/++$ & $-/+$ \\
\hline $\begin{array}{l}\text { Pontine nucleus } \\
\text { Pons }\end{array}$ & ++ & ++ & +/++ & +/++ \\
\hline Accessory facial nucleus & - & $-/+$ & + & $-/+$ \\
\hline Facial nucleus & $-/+$ & $-1+$ & + & + \\
\hline Dorsal cochlear nucleus & +/++ & + & ++ & + \\
\hline Nucleus of the solitary tract & + & $-1+$ & - & $-1+$ \\
\hline Hypoglossal nucleus & $-1+$ & $-1+$ & + & $-1+$ \\
\hline Cuneate nucleus & $-/+$ & $-/+$ & +/++ & $-/+$ \\
\hline Dorsal motor nucleus of vagus & $-1+$ & $-1+$ & - & $-1+$ \\
\hline Lateral reticular nucleus & $-1+$ & $-1+$ & $+/++$ & $-1+$ \\
\hline Gracile nucleus & - & $-1+$ & +/++ & - \\
\hline Inferior olive & - & + & $++/+++$ & + \\
\hline \multicolumn{5}{|l|}{ Cerebellum } \\
\hline Molecular layer & - & - & - & - \\
\hline Granular layer & +++ & +++ & +++ & +++ \\
\hline White matter & - & $-/+$ & ++ & $-/+$ \\
\hline \multirow{2}{*}{\multicolumn{5}{|c|}{ Circumventricular organs }} \\
\hline & & & & \\
\hline Choroid plexus & - & $-/+$ & - & - \\
\hline Area postrema & ++ & +/++ & $+/++$ & - \\
\hline White matter & - & - & $++/+++$ & + \\
\hline
\end{tabular}

${ }^{1} \mathrm{mRNA}$ data are expressed as semiquantitative estimates of hybridization intensity obtained by microdensitometric analysis of film autoradiograms. The levels of hybridization signals are: "+++" very strong; "++" moderate; "+" weak; "-/+" very weak and "-" not detected. "White matter" includes pyramidal tract, genu corpus callosum, cerebral peduncle and anterior commissure. 


\section{Figure legend}

Fig.1. Regional distribution of PDE4B splice variant mRNAs in rat brain. Film autoradiograms from rat rostral to caudal sections are presented, showing the hybridization pattern of PDE4B1 $\left(A_{1}-A_{6}\right)$, PDE4B2 $\left(B_{1}-B_{6}\right)$, PDE4B3 $\left(C_{1}-C_{6}\right)$, and PDE4B4 $\left(D_{1}-D_{6}\right)$. Note the strong hybridization signal of PDE4B3 in white matter, and the exclusive PDE4B2 expression in dentate gyrus of the hippocampus. aca, anterior commissure, anterior part; AcbSh, accumbens nucleus, shell; aci, anterior commissure, intrabulbar part; AO, anterior olfactory nucleus; AP, area postrema; Arc, arcuate nucleus; CA, Ammon's horn of hippocampus; chp, choroid plexus; cp, cerebral peduncle; CPu, caudate putamen (striatum); DCo, dorsal cochlear nucleus; DG, dentate gyrus; gcc, genu of the corpus callosum; hil, hilus; LRt, lateral reticular nucleus; ME, median eminence; MG: medial genicular nucleus; $\mathrm{MHb}$, medial habenular nucleus; 3, oculomotor nucleus; Pir, piriform cortex; Pn, pontine nuclei; PV, paraventricular thalamic nucleus; py, pyramidal tract; S1: primary somatosensory cortex. Bar $=3 \mathrm{~mm}$.

Fig. 2. Distribution of COX-2 mRNA in rat hippocampus from control (A) and LPS stimulated rats at $2 \mathrm{~h}$ post-injection (B). Note the profound transcriptional activation (visualized as a spotlike mRNA signal) detected over blood vessels of the entire brain microvasculature and leptomeninges of LPS-injected rats. Bar $=1 \mathrm{~mm}$. 
Figura 1

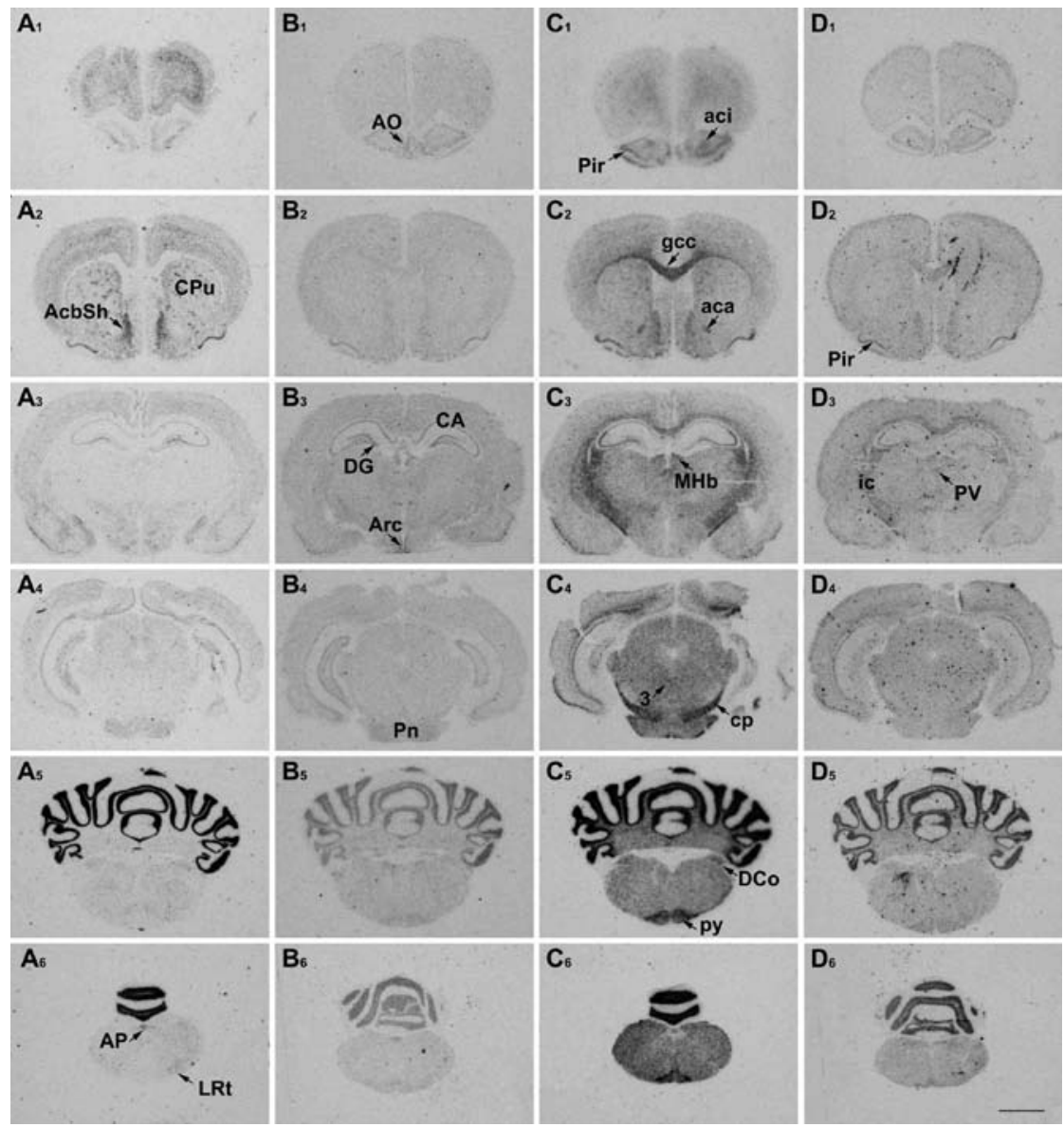

Figura 2

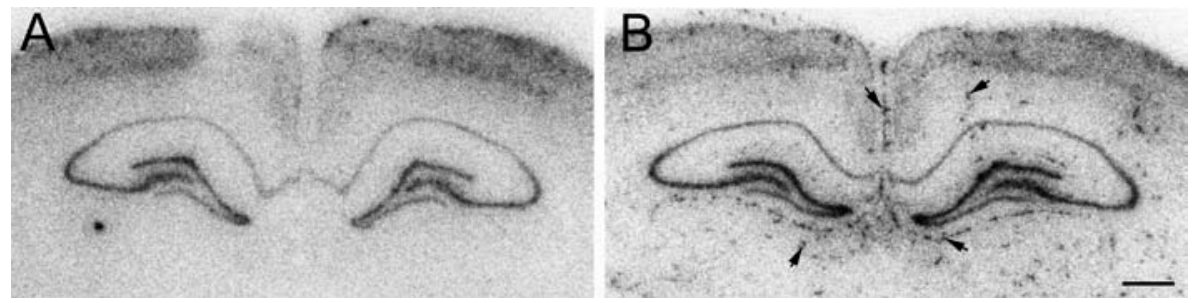

